\title{
Assessment of radiation doses and image quality of multiple low-dose CT exams in COVID-19 clinical management
}

\author{
Lu Bai ${ }^{1} \cdot$ Jie Zhou $^{2} \cdot$ Cong Shen ${ }^{1} \cdot$ Shubo $\mathrm{Cai}^{2} \cdot$ Youmin Guo ${ }^{1}$ Xunan Huang ${ }^{3} \cdot$ Guang Jia $^{3}$ (1) $\cdot$ Gang Niu $^{1}$
}

Received: 17 January 2021 / Revised: 22 August 2021 / Accepted: 9 September 2021 / Published online: 8 October 2021

(c) The Author(s), under exclusive licence to Springer Nature Singapore Pte Ltd. 2021

\begin{abstract}
Purpose The Corona Virus Disease 2019 (COVID-19) was first reported in December 2019 from an outbreak of unexplained pneumonia in Wuhan (Hubei, China) that subsequently spread rapidly around the world. Because of the public health emergency, chest CT has been widely used for sensitive detection and diagnosis, monitoring the changes of lesions and also for treatment evaluation. The purpose of this study was to investigate radiation dose and image quality of chest CT scans received by COVID-19 patients and to evaluate the oncogenic risk of multiple chest CT examinations.

Methods A retrospective review of 33 patients with RT-PCR confirmed COVID-19 infection was performed from January 31, 2020 to February 19, 2020. The date of each CT exam and respective radiation dose for each exam was recorded for all patients. Multiple pulmonary CT scans were obtained during diagnosis and treatment procedure. Scan frequency, total scan times, radiation dose, and image quality were determined.

Results Thirty-three patients (15 males and 18 females, age 21-82 years) with confirmed COVID-19 pneumonia underwent a total of 143 chest CT scans. The number of CT scans per patient was $4 \pm 1$, with a range of $2-6$. The time interval between two consecutive chest CT scans was $3 \pm 1$ days. The average effective dose from a single chest CT scan was $1.21 \pm 0.10 \mathrm{mSv}$, with a range of $1.02-1.44 \mathrm{mSv}$. The average cumulative effective dose per patient was $5.25 \pm 1.52 \mathrm{mSv}$, with a range of 2.24-7.48 mSv. The maximum cumulative effective dose was $7.48 \mathrm{mSv}$ for six CT examinations during COVID-19 treatment. Based on subjective image quality analysis, the visual scoring of CT findings was $11.23 \pm 1.35$ points out of 15 points. Conclusions The frequency, total number and image quality of chest CT scans should be reviewed carefully to guarantee minimally required CT scans during the COVID-19 management.
\end{abstract}

Keywords COVID-19 $\cdot$ CT $\cdot$ Radiation safety

\section{Introduction}

Guang Jia

gjia@xidian.edu.cn

$\triangle$ Gang Niu

niugang369@126.com.cn

1 Department of Medical Imaging, The First Affiliated Hospital of Xi' an Jiaotong University, Xi' an 710061, Shaanxi, China

2 Department of Radiology, Xi'an Chest Hospital, Xi'an, Shaanxi, China

3 School of Computer Science and Technology, Xidian University, No. 2 South Taibai Rd, Xi'an 710071, Shaanxi, China
The Corona Virus Disease 2019 (COVID-19) was first reported in December 2019 from an outbreak of unexplained pneumonia in Wuhan (Hubei, China) that subsequently spread rapidly around the world [1]. Because of the public health emergency, chest CT has been used for initial diagnosis, monitoring the changes of lesions and also for treatment evaluation [2]. Based on a survey with participants from 62 health care sites in 34 countries, $22 \%$ of sites used chest CT for initial diagnosis and $76 \%$ used chest $\mathrm{CT}$ to assess severity of COVID-19 pneumonia [3].

Diagnosis of suspected COVID-19 is often performed by detection of the viral nucleic acid via real-time polymerase chain reaction (RT-PCR) [4]. CT imaging is preferred because of high image contrast and sensitivity for detection or monitoring of COVID-19 viral pneumonia [5]. Some 
studies have reported symptomatic COVID-19 patients with viral pneumonia detected with CT imaging yet RT-PCR testing was negative $[2,6]$. Compared with chest radiography, CT scans can sensitively detect infection progression and patient's recovery from COVID-19 pneumonia [7], although few cases without any significant abnormalities in asymptomatic COVID-19 carriers were reported [8].

The purpose of this study was to investigate the image quality and radiation dose of low-dose CT scans received by COVID- 19 patients and to evaluate the oncogenic risk of multiple chest CT examinations.

\section{Materials and methods}

This retrospective study was approved by the National Health Commission of China and Ethics Commission of the First Affiliated Hospital of Xi' an Jiaotong University. Written informed consent was waived by the Ethics Commission of the designated hospital for emerging infectious diseases. Our study enrolled consecutive inpatients with RT-PCR confirmed COVID-19 infection between January 31, 2020 and February 19, 2020. The indication for chest CT was fever and suspected COVID-19 pneumonia. For each patient, one or multiple CT scans were performed at several time points during the course of the disease. The patients were scanned using low-dose protocol for a follow-up period of 2-3 weeks. The scan exclusion criteria included (a) critically severe COVID-19 cases and (b) children with COVID-19.

Chest CT scans were performed on a multi-detector CT scanner (Optima CT680, GE Healthcare, Waukesha, WI, USA). Images were acquired from the apex to base of the lungs during breath-hold for the inspiratory phase. The technical parameters for acquisition were $120 \mathrm{kVp}$ tube voltage, 120-380 mA tube current modulation, 10 noise index, 1.375 pitch, $0.8 \mathrm{~s}$ rotation time, and $4 \times 10 \mathrm{~mm}$ collimation. All images were reconstructed using filtered back projection algorithm and the STANDARD kernel at slice thickness of $1.25 \mathrm{~mm}$ and interval thickness of $1.25 \mathrm{~mm}$. The radiation output in the form of the dose-length product (DLP) was recorded from the CT system for each exam. The DLP was multiplied by a $\mathrm{k}$ factor of 0.014 for conversion to effective dose [9].

CT images of all patients were sent to the workstation for image analysis. The image quality of lung window and mediastinal window was scored by an experienced radiologist. The specific scoring criteria are as follows: (1) lung texture classification: clear, smooth and sharp edge, 3 points; unclear, unsmooth and sharp edge, 2 points; fuzzy edge, 1 point. (2) Image graininess: fine image with little graininess, 3 points; less fine image with slight graininess, 2 points; rough image with obvious graininess, 1 point. (3) The contrast of large mediastinal vessels: clear contrast, 3 points; unclear contrast, 2 points; fuzzy contrast, 1 point. (4) The clarity of tissue organization of mediastinal window: clear tissue organization, 3 points; unclear tissue organization, 2 points; fuzzy tissue organization level, 1 point. (5) Doctor's confidence in diagnosis: full confidence, 3 points; poor confidence, 2 points; lack of confidence 1 point. Overall visual scoring was calculated as the total points of all five criteria.

\section{Results}

Thirty-three patients (15 males and 18 females, age 21-82 years) with confirmed COVID-19 pneumonia underwent a total of 143 chest CT scans. The relevant diagnosis content as evaluated by an experienced radiologist is listed in Table 1. The number of CT scans per patient was $4 \pm 1$, with a range of $2-6$. Three patients (9\%) received two scans, six patients $(18 \%)$ received three scans, six patients (18\%) received four scans, 13 patients (39\%) received five scans, and five patients (15\%) received six scans.

The time interval between two consecutive chest CT scans was $3 \pm 1$ days, including 2-day intervals 11 exams (10\%), 3-day intervals 52 exams (47\%), 4-day intervals 35 exams (32\%), 5-day intervals 9 exams (8\%), and 6-day intervals 3 exams (3\%) (Fig. 1).

The average effective dose from a single chest CT scan was $1.21 \pm 0.10 \mathrm{mSv}$, with a range of $1.02-1.44 \mathrm{mSv}$. The average cumulative effective dose per patient was $5.25 \pm 1.52 \mathrm{mSv}$, with a range of 2.24-7.48 mSv (Fig. 2). The maximum cumulative effective dose was $7.48 \mathrm{mSv}$ for six CT examinations during COVID-19 treatment.

Based on subjective image quality analysis, the overall visual scoring of CT findings was $11.23 \pm 1.35$ out of 15 points (Table 2).

Table 1 CT image interpretations of patients with COVID-19

\begin{tabular}{lc}
\hline Pneumonia chest CT findings & $n(\%)$ \\
\hline GGO & $23(70 \%)$ \\
GGO pure & $22(67 \%)$ \\
GGO N-pure & $1(3 \%)$ \\
Consolidation & $5(15 \%)$ \\
Mixed GGO and consolidation & $0(0 \%)$ \\
Fibrous stripes & \\
Zonal predominance & $21(64 \%)$ \\
Upper & $30(91 \%)$ \\
Middle or lower
\end{tabular}

$G G O$ pure pure ground-glass opacities, GGO N-pure GGO with interlobular septal thickening 
Fig. 1 Chest CT scan frequency during COVID-19 clinical management. The range of time intervals between two consecutive chest CT scans was 2-6 days

\section{Time Intervals Between Two Scans}

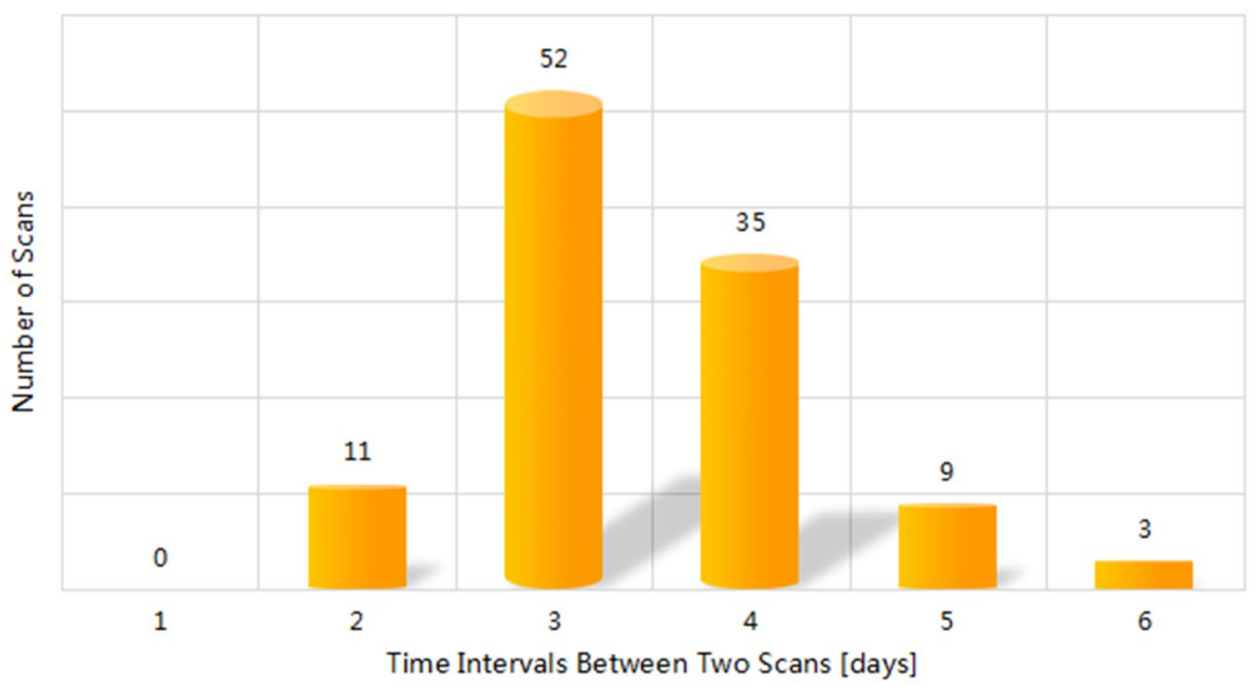

Effective Dose of Chest CT Scans

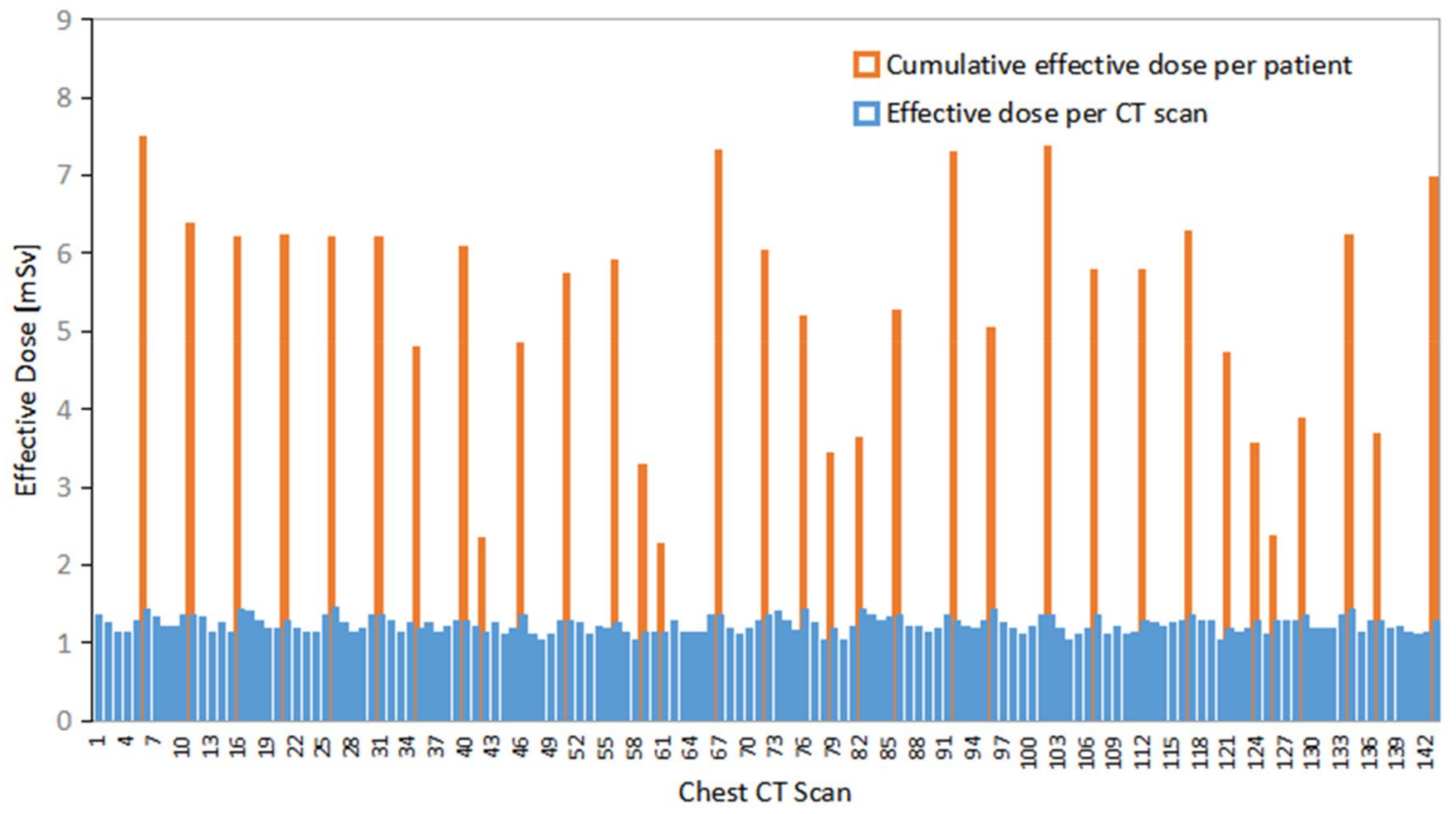

Fig. 2 Effective dose of chest CT scans. The effective dose of each chest CT scan (blue) was $1.21 \pm 0.10 \mathrm{mSv}$, and the cumulative effective dose of each patient (red) was $5.25 \pm 1.52 \mathrm{mSv}$

Table 2 Subjective evaluation of low-dose CT images

\begin{tabular}{lr}
\hline Evaluation index & \multicolumn{1}{l}{ Score } \\
\hline Lung texture classification & $2.27 \pm 0.45$ \\
Image graininess & $2.24 \pm 0.49$ \\
Contrast of large mediastinal vessels & $2.18 \pm 0.63$ \\
Clarity of tissue organization of mediastinal window & $2.31 \pm 0.50$ \\
Doctor's confidence in diagnosis & $2.21 \pm 0.69$ \\
Overall scoring & $11.23 \pm 1.35$ \\
\hline
\end{tabular}

\section{Discussion}

There are still big concerns about the net benefit of applying chest CT to COVID-19 management. The International Atomic Energy Agency (IAEA) organized a webinar to discuss CT practice and protocols for COVID-19 from a radiation protection perspective [10]. The median CT dose index for chest CT was 9 mGy in COVID-19 pneumonia 
as summarized from 782 patients from 54 health care sites in 28 countries, which has eightfold variations across multiple health care sites and is deemed as regular dose [11]. For example, Pan et al. reported CTDIvol of $8.4 \pm 2.0 \mathrm{mGy}$ for single chest CT exam of COVID-19 patients [7]. Lowdose CT ( $<3 \mathrm{mGy})$ has been proposed for the detection and management of COVID-19 using an improved detector, higher pitch settings, lower tube voltage and current, iterative reconstruction, and dose-reduction options [12]. An ultra low-dose $(<1 \mathrm{mGy})$ protocol exhibited highly diagnostic images for COVID-19 using fast, long-pitch and dual-source acquisition on third-generation dual-source CT scanners [13].

The development of low-dose CT imaging has unmet clinical needs as CT exams account for the major cause of radiation exposure from diagnostic imaging procedures [14]. In this study, the CT scan radiation dose to COVID19 patients was minimized through an improved detector, high-pitch setting, iterative reconstruction, and dosereduction options. The average effective dose for chest $\mathrm{CT}$ exams as reported in the literature is $10.3 \mathrm{mSv}$ and range of 3.2-12.2 $\mathrm{mSv}$ [15]. In this study, the average effective dose for a single CT scan was $1.21 \mathrm{mSv}$, which can be deemed as a low-dose protocol [13] and associated with an oncogenic risk of $0.006 \%$ [16]. The average total effective dose per patient with multiple CT exams was $5.25 \mathrm{mSv}$ in this study, which would result in approximately $0.03 \%$ additional lifetime risk for fatal cancer.

Several studies have evaluated the role of low-dose CT for COVID-19 management. Low-dose CT imaging protocol may result in the loss of image quality and could potentially affect clinical diagnosis of COVID-19 [13, 17, 18]. In this study, the overall scoring assigned by the radiologist showed an acceptance rate of 11.23 out of 15 .

We are aware that our study has some limitations. This is a single-center study. Both radiation dose and image quality of the specific CT protocol could not be strictly compared to other institutions. It would be better to scan same patients with both low-dose protocol and standarddose protocol for a subjective comparison [12]. Another limitation of this retrospective study is that the scan protocol has not been systematically optimized. For example, $120 \mathrm{kVp}$ was used in contrary to the suggested low tube voltage $(80-100 \mathrm{kVp})$ [19]. In future studies, tube voltage and current, pitch setting, and reconstruction method should be evaluated for minimal dose and high image quality. In this study, a small amount of patients was enrolled within a short period of time, thus the role of CT scan in COVID-19 management could not be thoroughly defined. Further evaluation of the value of low-dose CT scan in COVID-19 will require studies with large sample sizes and long-term follow-up.

\section{Conclusions}

This study illustrates there is evidence to optimize protocols and reduce the radiation exposure per exam such that the cumulative radiation exposure from multiple CT exams is reduced. Furthermore, the frequency and total number of chest $\mathrm{CT}$ scans should be reviewed carefully to guarantee minimally required $\mathrm{CT}$ scans during the treatment to follow the ALARA (As Low As Reasonably Achievable) principal.

Acknowledgements We thank Joe Steiner, $\mathrm{PhD}$ (Department of Radiology, Henry Ford Health System, Detroit MI) and Rick Layman, PhD, DABR (Department of Imaging Physics, MD Anderson Cancer Center, Houston TX) for data interpretation and revisions. None of these persons received any compensation for their contributions.

Author contributions GN and GJ had full access to all of the data in the study and take responsibility for the integrity of the data and the accuracy of the data analysis. GN and GJ contributed equally to the work. Concept and design: GN, GJ, and LB. Acquisition, analysis, or interpretation of data: LB, JZ, CS, XH, GJ, and GN. Drafting of the manuscript: LB, XH, GJ, and GN. Critical revision of the manuscript for important intellectual content: LB, XH, GJ, and GN. Statistical analysis: LB, GJ, and YG. Administrative, technical, or material support: LB, SC, XH, GJ, and GN. Supervision: GN and GJ.

Funding This work was supported by National Natural Science Foundation of China (No. 11974267), Science and Technology Planning Project of XI'AN (No. GXYD6.2) and National Natural Science Foundation of China (No. 61771369).

\section{Declarations}

Conflict of interest On behalf of all the authors, the corresponding author states that there is no conflict of interest.

Ethics approval The study protocol was complied with the principles of the Declaration of Helsinki and was approved by the National Health Commission of China and Ethics Commission of the First Affiliated Hospital of Xi' an Jiaotong University.

Consent to participate Written informed consent was waived by the Ethics Commission of the designated hospital for emerging infectious diseases.

Consent for publication Not applicable.

\section{References}

1. Paules CI, Marston HD, Fauci AS. Coronavirus infections-more than just the common cold. JAMA. 2020;323:707-8. https://doi. org/10.1001/jama.2020.0757.

2. Fang Y, Zhang H, Xie J, Lin M, Ying L, Pang P, Ji W. Sensitivity of chest CT for COVID-19: comparison to RT-PCR. Radiology. 2020;296:E115-7. https://doi.org/10.1148/radiol.2020200432.

3. Homayounieh F, Holmberg O, Umairi RA, Aly S, Basevičius A, Costa PR, et al. Variations in CT utilization, protocols, and radiation doses in COVID-19 pneumonia: results from 28 countries in the IAEA study. Radiology. 2021;298:E141-51. https://doi.org/ 10.1148/radiol.2020203453. 
4. Chung M, Bernheim A, Mei X, Zhang N, Huang M, Zeng X, et al. CT Imaging features of 2019 novel coronavirus (2019-nCoV). Radiology. 2020;295:202-7. https://doi.org/10.1148/radiol.20202 00230.

5. Wang YXJ, Liu W-H, Yang M, Chen W. The role of CT for Covid19 patient's management remains poorly defined. Ann Trans Med. 2020;8:145. https://doi.org/10.21037/atm.2020.02.71.

6. Xie X, Zhong Z, Zhao W, Zheng C, Wang F, Liu J. Chest CT for typical coronavirus disease 2019 (COVID-19) pneumonia: relationship to negative RT-PCR testing. Radiology. 2020;296:E41-5. https://doi.org/10.1148/radiol.2020200343.

7. Pan F, Ye T, Sun P, Gui S, Liang B, Li L, et al. Time course of lung changes at chest $\mathrm{CT}$ during recovery from coronavirus disease 2019 (COVID-19). Radiology. 2020;295:715-21. https://doi. org/10.1148/radiol.2020200370.

8. Bai Y, Yao L, Wei T, Tian F, Jin D-Y, Chen L, Wang M. Presumed asymptomatic carrier transmission of COVID-19. JAMA. 2020;323:1406-7. https://doi.org/10.1001/jama.2020.2565.

9. Christner JA, Kofler JM, McCollough CH. Estimating effective dose for CT using dose-length product compared with using organ doses: consequences of adopting International Commission on Radiological Protection publication 103 or dual-energy scanning. Am J Roentgenol. 2010;194:881-9. https://doi.org/10.2214/AJR. 09.3462.

10. Kalra MK, Homayounieh F, Arru C, Holmberg O, Vassileva J. Chest CT practice and protocols for COVID-19 from radiation dose management perspective. Eur Radiol. 2020;30:6554-60. https://doi.org/10.1007/s00330-020-07034-x.

11. Azadbakht J, Khoramian D, Lajevardi ZS, Elikaii F, Aflatoonian $\mathrm{AH}$, Farhood B, et al. A review on chest CT scanning parameters implemented in COVID-19 patients: bringing low-dose CT protocols into play. Egypt J Radiol Nucl Med. 2021. https://doi.org/ 10.1186/s43055-020-00400-1.

12. Kang Z, Li X, Zhou S. Recommendation of low-dose CT in the detection and management of COVID-2019. Eur Radiol. 2020;30:4356-7. https://doi.org/10.1007/s00330-020-06809-6.

13. Agostini A, Floridi C, Borgheresi A, Badaloni M, Esposto Pirani P, Terilli F, et al. Proposal of a low-dose, long-pitch, dual-source chest CT protocol on third-generation dual-source $\mathrm{CT}$ using a tin filter for spectral shaping at $100 \mathrm{kVp}$ for CoronaVirus Disease 2019 (COVID-19) patients: a feasibility study. Radiol Med (Torino). 2020;125:365-73. https://doi.org/10.1007/ s11547-020-01179-x.

14. Shiri I, Akhavanallaf A, Sanaat A, Salimi Y, Askari D, Mansouri Z, et al. Ultra-low-dose chest CT imaging of COVID-19 patients using a deep residual neural network. Eur Radiol. 2021;31:1420 31. https://doi.org/10.1007/s00330-020-07225-6.

15. Gorycki T, Lasek I, Kamiński K, Studniarek M. Evaluation of radiation doses delivered in different chest CT protocols. Pol J Radiol. 2014;79:1-5. https://doi.org/10.12659/PJR.889952.

16. Vilar-Palop J, Vilar J, Hernández-Aguado I, González-Álvarez I, Lumbreras B. Updated effective doses in radiology. J Radiol Protect Off J Soc Radiol Protect. 2016;36:975-90. https://doi.org/ 10.1088/0952-4746/36/4/975.

17. Radpour A, Bahrami-Motlagh H, Taaghi MT, Sedaghat A, Karimi MA, Hekmatnia A, et al. COVID-19 evaluation by low-dose high resolution CT scans protocol. Acad Radiol. 2020;27:901. https:// doi.org/10.1016/j.acra.2020.04.016.

18. Dangis A, Gieraerts C, de Bruecker Y, Janssen L, Valgaeren H, Obbels D, et al. Accuracy and reproducibility of low-dose submillisievert chest CT for the diagnosis of COVID-19. Radiol Cardiothorac Imaging. 2020;2: e200196. https://doi.org/10.1148/ryct. 2020200196.

19. de Margerie-Mellon C, de Bazelaire C, Montlahuc C, Lambert $\mathrm{J}$, Martineau A, Coulon $\mathrm{P}$, et al. Reducing radiation dose at chest CT: comparison among model-based type iterative reconstruction, hybrid iterative reconstruction, and filtered back projection. Acad Radiol. 2016;23:1246-54. https://doi.org/10.1016/j.acra.2016.05. 019 .

Publisher's Note Springer Nature remains neutral with regard to jurisdictional claims in published maps and institutional affiliations. 